\title{
Gradually worsening dyspnea and dry cough in an elderly patient
}

\author{
Emanuela Barisione1, Giovanni A. Rossi2, Mario Salio1 \\ 1 Ospedale Policlinico San Martino, Genoa \\ 2 Pulmonary and Allergy Disease Unit and Cystic Fibrosis Center, Istituto Giannina Gaslini, Genoa, Italy
}

\begin{abstract}
Primary tracheal chondrosarcomas are an extremely rare condition that affect adult of all ages, $24 \%$ being more that 70 years old. The progression may be slow, with symptoms that may continue up to 72 months before diagnosis is made. The clinical manifestations, that include a combination of nonproductive cough, wheezing, and dyspnea without hemoptysis often leads to misdiagnosis of asthma or chronic obstructive pulmonary disease. We report a case of an 87-year-old male, a Medical Doctor, with such a history, in whom a diagnosis of a tracheal chondrosarcoma was finally performed, 12 months after the first manifestation of the disease.
\end{abstract}

\section{Introduction}

Asthma is a disorder whose symptoms typically appear in childhood or in young adulthood [1]. When these occur late in life and there is no prompt response to treatment is mandatory to consider other diagnoses such as heart failure, gastroesophageal reflux and other forms of airway obstruction [2]. Especially in the elderly, when symptoms are mild with a slow progression, and the clinical history, the physical examination, the chest X-ray and the spirometry do not suggest an alternative diagnosis, more invasive diagnostic tests are deferred in time, waiting for a possible spontaneous healing [3].

Corresponding Author: Giovanni A. Rossi, Pulmonary and Allergy Disease Unit and Cystic Fibrosis Center, Istituto Giannina Gaslini, Largo G. Gaslini, 4. 16148 Genoa, Italy. Fax. +39.10.3760013. E-mail: giovannirossi@gaslini.org

Key words: Chondrosarcoma; tracheal obstruction; bronchoscopy; laser; tumor.

Conflict of interest: The authors declare no conflict of interest.

Received for publication: 26 April 2017

Accepted for publication: 1 July 2017

(C) Copyright E. Barisione et al., 2017

Tipografia PI-ME Editrice, Italy

Monaldi Archives for Chest Disease 2017; 87:834

doi: 10.4081/monaldi.2017.834

This article is distributed under the terms of the Creative Commons Attribution Noncommercial License (by-nc 4.0) which permits any noncommercial use, distribution, and reproduction in any medium, provided the original author(s) and source are credited.

\section{Case report}

An 87-year-old man, ex-smoker (he had stopped 25 years before) presented with dry cough and a slowly gradually worsening exertional dyspnea. One year before, when he began to have episodes of cough, he was firstly evaluated by a General practitioner: the chest Xray showed an increased pulmonary transparency, at least partly due to reduction of the parietal muscle thickness, a 'barrel chest' and bronchial wall thickening, whilst the pulmonary function tests demonstrated a mild obstructive pattern, with a modest response to salbutamol. He was diagnosed as chronic obstructive pulmonary disease, and an inhaled anticholinergic drug, tiotropium bromide, was recommended. His condition improved to some extent only for a short period. Bronchodilators, inhaled and systemic corticosteroids were prescribed with no response and, lately, dyspnea was associated with mild inspiratory stridor and wheezing at maximal exertion. At admission, physical examination showed poor general condition and decreased breath sounds with light stridor and wheeze, at chest auscultation, when the patient was breathing fast and deeply. 0xygen saturation $\left(\mathrm{SaO}_{2}\right)$ at rest, in room air, was $94 \%$ and no signs of congestive heart failure were detected. The chest x-ray did not show any change, as compared to the previous one, whist spirometry demonstrated the flattening of the expiratory limb of the flow/volume loop, consistent with the presence of a variable intrathoracic obstruction. A chest computed tomography (CT) scan showed an endoluminal mass arising from the right lateral wall of the trachea, nearly completely occluding the lumen (Figure 1A). A rigid bronchoscopy was performed (Efer-Dumon Novatech ${ }^{\circledR}$, La Ciotat, France) in general anesthesia: a lobulated, hypervascular mass was detected (Figure 1B), that was biopsied and partially removed with laser assisted mechanical resection. Histologically, the mass was characterized as a partially calcified low-grade tracheal chondrosarcoma: cytological findings included round or polygonal cells with occasional binucleation, round hyperchromatic nuclei and prominent nucleoli, present in an amorphous pink-violet or light-blue background containing fragments of chondroid tissue. There was no indication for surgical resection for the mediastinal involvement and for the compromised cardiovascular conditions of the old patient. A slowly growing local recurrence was observed in the follow-up visit four month later, that did not require a second endoscopic resection. The patient died ten months after for heart failure.

\section{Discussion}

Primary tracheal tumors show a incidence of approximately 0.1 in every 100,000 persons per year, squamous cell carcinomas and adenoid cystic carcinomas accounting for about two-thirds of them, whereas chondrosarcoma is an exceptional occurrence with only 24 cases described so far [4-7]. The mean age of the patients reported 

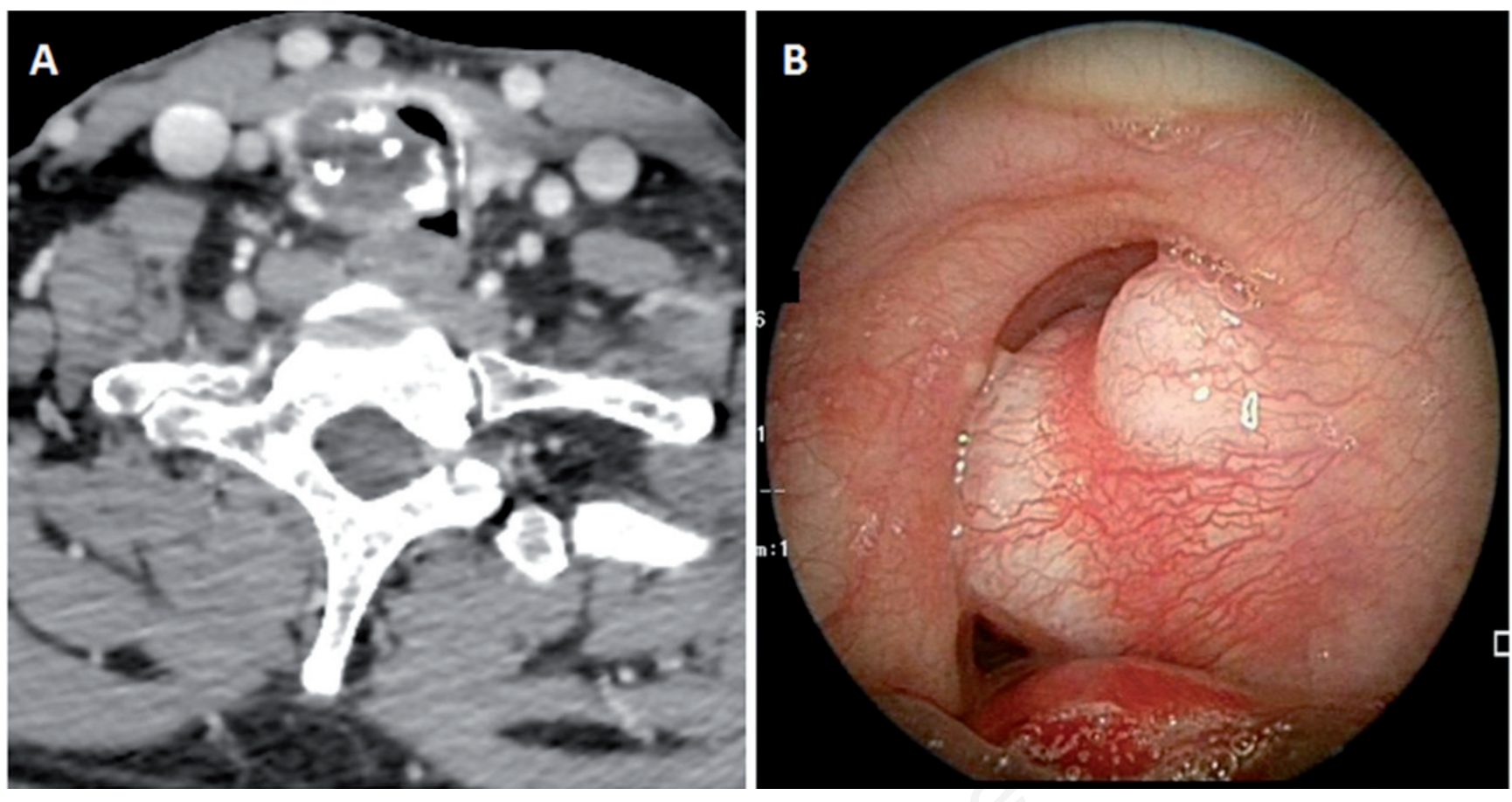

Figure 1. A) CT scan (mediastinal window) showing a partially calcified endotracheal mass protruded into the trachea, resulting in a $>90 \%$ reduction of the cross-sectional area. B) Bronchoscopic view of the lobulated, hypervascular mass, nearly completely occluding the tracheal lumen.

was 62.7 years, the youngest being 32 years old and 10 of them being above 70 years of age. For unknown reasons all but one were males. Based on cellularity, atypia, and pleo-morphism, chondrosarcomas can be classified into 3 histologic grades: I, II and III. Low-grade (III) chondrosarcomas tend to grow slowly and, when located in the airways, in general do not metastasize [6]. Because of their slow growth, tracheal condrosarcomas are often misdiagnosed: dyspnea, cough and wheezing are the most common reported symptoms, often leading to a wrong diagnosis of difficult-to treat asthma or of recurrent chronic obstructive pulmonary disease exacerbations [1-3]. As in our patient, spirometry can suggest the presence of a variable intrathoracic obstruction $[8,9]$. CT scan and flexible bronchoscopy with forceps biopsies are the essential methods for diagnosis and followup, whilst rigid bronchoscopy is mandatory before surgery, both for diagnostic and therapeutic purposes [6,7,9]. The optimal treatment is tracheal sleeve-resection with end-to-end anastomosis, with or without initial laser debulking [10]. Incomplete surgical resection (positive surgical margins or a 'debulking' procedure) or endoscopic resection only are considered palliative options to restore an adequate airway patency in emergency conditions or in inoperable patients or as the initial definitive treatment [10]. Although successful treatment with radiotherapy has been reported, the well-differentiated tracheal condrosarcomas is thought to be unresponsive to chemo and radiotherapy [11].

\section{Conclusions}

Asthmatic symptoms, occurring in adulthood (and chiefly in elderly people), and no response to treatment should suggest to consider other diagnoses. Despite the old age of the patient, other diagnostic tests in addition to the chest X-ray and the spirometry should be planned and not deferred when respiratory conditions are deteriorating.

\section{References}

1. Global Strategy for Asthma Management and Prevention, Global Initiative for Asthma (GINA) 2017. Available from: http//ginasthma. org/gina-reports.

2. Miravitlles M, Andreu I, Romero Y, et al. Difficulties in differential diagnosis of COPD and asthma in primary care. Br J Gen Pract 2012; 62:e68-75.

3. Sano H, Iwanaga T, Nishiyama 0 , et al. Characteristics of phenotypes of elderly patients with asthma. Allergol Int 2016;65:204-9.

4. Honings J, van Dijck JA, Verhagen AF, et al. Incidence and treatment of tracheal cancer: a nationwide study in the Netherlands. Ann Surg Oncol 2007;14:968-76.

5. Macchiarini P. Primary tracheal tumours. Lancet Oncol 2006;7: 83-91.

6. Maia D, Elharrar X, Laroumagne S, et al. Malignant transformation of a tracheal chondroma: The second reported case and review of the literature. Rev Port Pneumol 2016;22:283-6.

7. Scherl S, Alon EE, Karle WE, et al. Rare tracheal tumors and lesions initially diagnosed as isolated differentiated thyroid cancers. Thyroid 2013;23:79-83.

8. Lin DA, Arm JP. Asthma or not? The value of flow volume loops in evaluating airflow obstruction. Allergy Asthma Proc 2003;24:107-10.

9. Maish M, Vaporciyan AA. Chondrosarcoma arising in the trachea: a case report and review of the literature. J Thorac Cardiovasc Surg 2003;126:2077-80.

10. Andolfi M, Vaccarili M, Crisci R, Puma F. Management of tracheal chondrosarcoma almost completely obstructing the airway: a case report. J Cardiothorac Surg 2016;11:101.

11. Li Y, Peng A, Yang X, et al. Clinical manifestation and management of primary malignant tumors of the cervical trachea. Eur Arch Otorhinolaryngol 2014;271:225-35. 